\title{
OPTICAL PHOTOMETRIC MONITORING OF LS I +61 303*
}

\author{
X. PAREDES-FORTUNY ${ }^{\dagger}$, M. RIBÓ, O. FORS, J. NÚNẼZ and V. BOSCH-RAMON \\ Departament d'Astronomia i Meteorologia, Institut de Ciènces del Cosmos, \\ Universitat de Barcelona, IEEC-UB, Martí i Franquès 1, \\ E-08028 Barcelona, Spain \\ ${ }^{\dagger}$ xparedes@am.ub.es
}

Received 30 October 2013

Published 21 March 2014

\begin{abstract}
Three gamma-ray binaries, namely PSR B1259-63, HESS J0632+057 and LS I +61 303, contain compact objects orbiting around massive Be stars. Around periastron passage the compact objects should produce significant changes in the structure of the Be disks due to gravitational forces and eventually by ram pressure from the putative pulsar wind. Indeed, variability in the $\mathrm{H} \alpha$ emission line has been detected in all these systems, and optical periodic variability has been detected in one of them. However, there is lack of a systematic monitoring with accurate photometry, which could be used to constrain the shape of the disk during the periastron passage. This information is important to build accurate physical models to explain the broadband spectral energy distribution of these sources. Here we present an ongoing program to monitor the optical photometry of gamma-ray binaries and show preliminary results for the case of LS I +61 303 .
\end{abstract}

Keywords: Binaries: close; gamma rays: stars; stars: emission-line, Be.

PACS Numbers: 95.85.Kr, 97.80.Jp, 97.80.Fk

\section{Introduction}

Gamma-ray binaries are systems that comprise a young massive star and a compact object that display non-thermal emission dominated by $\mathrm{MeV}-\mathrm{GeV}$ photons. Five gamma-ray binaries are currently known. The nature of the compact object is only known in the case of PSR B1259-63, but the other systems could also contain young non-accreting pulsars with relativistic winds. Most, if not all, of these sources have been detected up to TeV energies. The spectral and brightness properties appear to be synchronised with the orbit of the binary system, and they do not show evidence of the presence of an accretion disk.

* This is an Open Access article published by World Scientific Publishing Company. It is distributed under the terms of the Creative Commons Attribution 3.0 (CC-BY) License. Further distribution of this work is permitted, provided the original work is properly cited. 
Two of the five known gamma-ray binaries, LS 5039 and 1FGL J1018.65856, have an O6 massive companion. The other three binaries, LS I +61 303, HESS J0632+057 and PSR B1259-63 have a Be massive companion with a circumstellar disk in the equatorial plane. See Ref. 1 and references therein.

In a gamma-ray binary containing a Be star the circumstellar disk is perturbed around periastron passage, due to both the gravitational forces induced by the compact object and the ram pressure of the expected relativistic pulsar wind.

The main goal of this observational project is to obtain and analyze very precisely optical photometric data to model the disk properties and obtain physical parameters of the systems. Here we present preliminary results on the optical photometric monitoring of the Be gamma-ray binary LS I +61 303. Preliminary results on the gamma-ray binary candidate HD 215227 were presented in Ref. 2.

\section{LS I +61 303}

LS I +61 303 is a gamma-ray binary composed by an optical star $(V \sim 10.8)$ with spectral type B0 Ve and a compact companion orbiting in a highly eccentric orbit with a period of 26.4960 day known from decades of radio outburst data. ${ }^{3}$ In Ref. 4 it was found an orbital solution that supports an eccentricity of $e=0.72 \pm 0.15$.

Variable flux modulated with the orbital period has been detected in $\mathrm{TeV},{ }^{5}$ $\mathrm{X}$-rays, optical photometry, $\mathrm{H} \alpha$ spectroscopy, and radio (see Ref. 3 and references therein). The distance to the source is estimated to be $\sim 2 \mathrm{kpc}$ based on HI measurements. ${ }^{6}$

Optical variability was detected (see Ref. 7), and a $26.5 \mathrm{~d}$ optical periodicity was suggested in Ref. 8. A period of $\sim 26 \mathrm{~d}$ in the $V$ band, and high resolution $\mathrm{H} \alpha$ spectra that may exhibit also periodic variability, were presented in Ref. 9. In Ref. 4 the orbital ephemeris were estimated, and the radio lightcurve maxima was found at $\sim 0.3-0.4$ orbital phases passed periastron, which takes place at orbital phase 0.23 .

\section{Telescope Fabra ROA Montsec}

The optical observations of this project are currently made with the robotic telescope Telescope Fabra-ROA Montsec (TFRM; see Ref. 10). The TFRM is installed at the Observatori Astronòmic del Montsec (Sant Esteve de la Sarga, Lleida, Spain).

The main specifications are: corrector plate of $0.5 \mathrm{~m}$ aperture and $0.78 \mathrm{~m}$ primary mirror, refurbished Baker-Nunn Camera for routine CCD robotic observations, focal ratio $\mathrm{f} / 0.96,4.4^{\circ} \times 4.4^{\circ}$ field of view with a pixel scale of $3.9^{\prime \prime} /$ pixel, passband filter SCHOTT GG475 ( $\lambda>475 \mathrm{~nm}$.), and CCD quantum efficiency of $60 \%$ at $550 \mathrm{~nm}$.

\section{Observational Strategy, Data Reduction and Analysis}

When possible, the Be gamma-ray binary LS I +61 303 is being observed around 20 times per night with exposures of $\sim 5$ s each. 
The data reduction and analysis is made using a pipeline developed in Python following these steps: calibration of the images using PyRAF, astrometric reduction using Astrometry.net, aperture photometry using SExtractor, and correction of the lightcurves using a mean differential magnitude correction method based on the principles of the BESTRED pipeline. ${ }^{11}$ See Ref. 2 for details.

\section{TFRM Observations of LS I +61303}

Here we present preliminary results on the ongoing program on LS I +61303 . The observations span from 2012 July 30 to 2013 February 16, thus covering seven orbital cycles of $26.5 \mathrm{~d}$, with 49 nights of good data.

The preliminary lightcurve of nightly averages is shown in Fig. 1-top. The folded lightcurve in Fig. 1-bottom shows a broad maximum between orbital phase 0.5 and
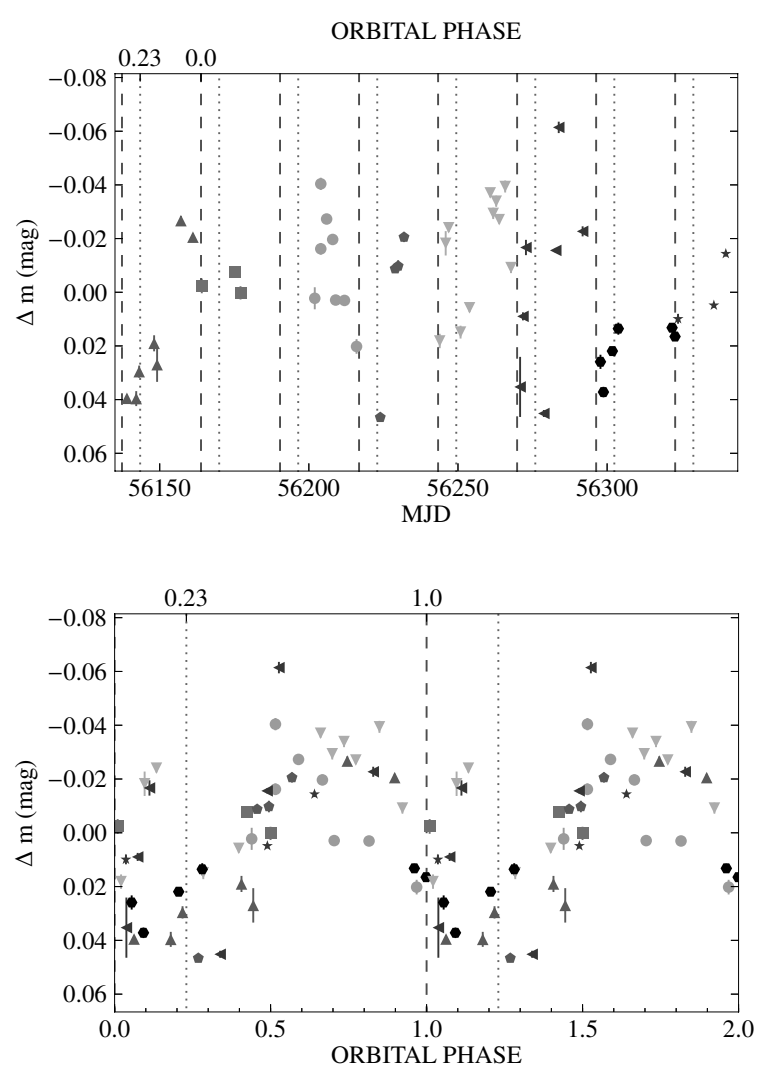

Fig. 1. Top: Lightcurve of the gamma-ray binary LS I +61 303 plotted as a function of MJD with an artificial offset to a mean magnitude of 0 . The data are binned on a nightly basis. Different symbols represent each cycle starting at phase 0.0. Periastron takes place at orbital phase 0.23 (dotted lines). Bottom: Lightcurve plotted as a function of the orbital phase for an orbital period of $26.4960 \mathrm{~d}$ and phase zero at JD 2,443,366.775. Two cycles are displayed for clarity. 


\section{Paredes-Fortuny et al.}

0.9 , thus around apastron passage, and a broad minimum in the phase range 0.0 0.4 , around periastron. Variability from cycle to cycle, reported here for the first time, is clear in Fig. 1-top. The results presented in Ref. 9, covering sparsely a time interval of $8 \mathrm{yr}$, show a large scatter, probably as a result of this cycle to cycle variability superimposed on eventual long-term trends related to the Be star.

Considering that the optical flux is basically coming from the Be star photosphere and the decretion disk, we can try to relate the periodic behavior of our data to periodic changes in the decretion disk. From the folded lightcurve we obtain a magnitude difference from maximum to minimum of $\Delta m \simeq 0.08 \mathrm{mag}$. Using the formula $m=-2.5 \log F+C$ we obtain a variation in flux of $\Delta F \simeq 0.07 F_{\text {total }}$. In Ref. 4 it was estimated that the emission of the disk represents a $35 \%$ of the total flux. If the observed variation only comes from the disk it implies $\Delta F \simeq 0.20$ $F_{\text {disk }}$. Assuming that there are no changes in the disk temperature, this flux change implies a variabiliy in the emitting area of a $20 \%$ as well. The folded lightcurve suggests that the disk emission (and area) is significantly reduced before periastron passage and increases slowly towards apastron, which might be related to the dynamical interaction between the pulsar wind and the stellar disk. We also note that the cycle to cycle variability and the observed night to night variability amount to $\sim 0.02 \mathrm{mag}$, implying changes of the disk emission (and area) of a $5 \%$.

\section{Conclusions}

The preliminary optical photometry of LS I +61 303 shows: the known periodic variability, variations in the lightcurve from cycle to cycle, and variations in magnitude compatible with a change of the disk emission (and area) of a $\sim 20 \%$ for the orbital modulation (increasing towards apastron), and of a $\sim 5 \%$ for the nightly changes and for the cycle to cycle changes.

\section{Acknowledgments}

The authors acknowledge support of the TFRM team for preparing and carrying out the optical observations. X.P.-F., M.R. and V.B-R. acknowledge support by the Spanish Ministerio de Economía y Competitividad under grants FPA2010-22056C06-02 and AYA2010-21782-C03-01. X.P.-F. also acknowledges financial support from Universitat de Barcelona under grant APIF. V.B-R. also acknowledges financial support from MINECO through a Ramón y Cajal fellowship. This research has been supported by the Marie Curie Career Integration Grant 321520.

\section{References}

1. J., Moldón, M., Ribó and J. M., Paredes, A\&A, 548, A103 (2012).

2. X., Paredes-Fortuny, M., Ribó, O., Fors and J., Núñez, American Institute of Physics Conference Series, 1505, 390 (2012).

3. P. C., Gregory, ApJ, 575, 427 (2002). 
4. J., Casares, I., Ribas, J. M., Paredes, J., Martí and C., Allende Prieto, MNRAS, 360, 1105 (2005).

5. J., Albert, E., Aliu, H., Anderhub, et al., ApJ, 693, 303 (2009).

6. D. A., Frail and R. M., Hjellming, AJ, 101, 2126 (1991).

7. J. M., Paredes and F., Figueras, A\&A, 154, L30 (1986).

8. H., Mendelson and T., Mazeh, MNRAS, 239, 733 (1989).

9. J. M., Paredes, P., Marziani, J., Marti, et al., A\&A, 288, 519 (1994).

10. O., Fors, J., Núñez, J., L. Muiños, et al., PASP, 125, 522 (2013).

11. H., Voss, Ph.D. Thesis, University of Berlin (2006). 